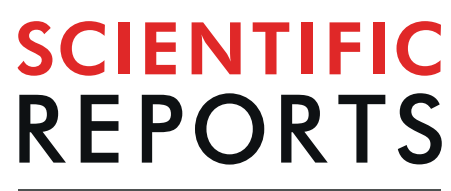

natureresearch

Check for updates

\title{
Growth Differentiation Factor 15 is a potential biomarker of therapeutic response for TK2 deficient myopathy
}

Cristina Dominguez-Gonzalez ${ }^{1,2,3}$, Carmen Badosa ${ }^{4}$, Marcos Madruga-Garrido ${ }^{5}$, Itxaso Martí ${ }^{6}$, Carmen Paradas ${ }^{7,8}$, Carlos Ortez ${ }^{4}$, Jordi Diaz-Manera ${ }^{3,9}$, Andres Berardo ${ }^{10}$, Jorge Alonso-Pérez ${ }^{9}$, Selena Trifunov ${ }^{4}$, Daniel Cuadras ${ }^{11}$, Susana G. Kalko ${ }^{12}$, Cora BlázquezBermejo ${ }^{3,13}$, Yolanda Cámara 3,13 , Ramon Martí, ${ }^{3,13}$, Fabiola Mavillard ${ }^{7,8}$, Miguel A. Martin ${ }^{2,3}$, Julio Montoya ${ }^{3,14}$, Eduardo Ruiz-Pesini $i^{3,14}$, Joan Villarroya ${ }^{15,16}$, Raquel Montero ${ }^{3,17}$, Francesc Villarroya ${ }^{15,16}$, Rafael Artuch ${ }^{3,17}$, Michio Hirano ${ }^{10}{ }^{\text {, Andrés Nascimento }}{ }^{3,4}$ \& Cecilia Jimenez-Mallebrera ${ }^{3,4,18 \bowtie ~}$

GDF-15 is a biomarker for mitochondrial diseases. We investigated the application of GDF-15 as biomarker of disease severity and response to deoxynucleoside treatment in patients with thymidine kinase 2 (TK2) deficiency and compared it to FGF-21. GDF-15 and FGF-21 were measured in serum from 24 patients with TK2 deficiency treated 1-49 months with oral deoxynucleosides. Patients were grouped according to age at treatment and biomarkers were analyzed at baseline and various time points after treatment initiation. GDF-15 was elevated on average 30 -fold in children and 6-fold in adults before the start of treatment. There was a significant correlation between basal GDF-15 and severity based on pretreatment distance walked (6MWT) and weight (BMI). During treatment, GDF15 significantly declined, and the decrease was accompanied by relevant clinical improvements. The decline was greater in the paediatric group, which included the most severe patients and showed the greatest clinical benefit, than in the adult patients. The decline of FGF-21 was less prominent and consistent. GDF-15 is a potential biomarker of severity and of therapeutic response for patients with TK2 deficiency. In addition, we show evidence of clinical benefit of deoxynucleoside treatment, especially when treatment is initiated at an early age.

${ }^{1}$ Neurology Department, Neuromuscular Disorders Unit, 12 de Octubre Hospital, Madrid, Spain. ${ }^{2}$ Research Institute $\mathrm{i}+12,12$ de Octubre Hospital, Madrid, Spain. ${ }^{3}$ Biomedical Network Research Centre on Rare Diseases (CIBERER), Instituto de Salud Carlos III, Madrid, Spain. ${ }^{4}$ Neuromuscular Unit, Neuropediatrics Department, Institut de Recerca Sant Joan de Déu, Hospital Sant Joan de Déu, Barcelona, Spain. ${ }^{5}$ Neuromuscular Disorders Unit, Neuropediatrics Department, Instituto de Biomedicina de Sevilla, Hospital Universitario Virgen del Rocío, Consejo Superior de Investigaciones Científicas, University of Seville, Seville, Spain. ${ }^{6}$ Neuropediatrics Department, Hospital Universitario Donostia, San Sebastian, Spain. ${ }^{7}$ Neuromuscular Disorders Unit, Neurology Department, Instituto de Biomedicina de Sevilla, Hospital Universitario Virgen del Rocío, Consejo Superior de Investigaciones Científicas, University of Seville, Seville, Spain. ${ }^{8}$ Biomedical Network Research Centre on Neurodegenerative Diseases (CIBERNED), Instituto de Salud Carlos III, Madrid, Spain. ${ }^{9}$ Neuromuscular Diseases Unit, Hospital de la Santa Creu i Sant Pau, Universitat Autònoma de Barcelona, Barcelona, Spain. ${ }^{10}$ Department of Neurology, Columbia University Medical Center, New York, USA. ${ }^{11}$ Statistics Unit, Fundación Sant Joan de Déu, Barcelona, Spain. ${ }^{12}$ Moebius Research Ltd, Systems Biomedicine, London, UK. ${ }^{13}$ Research group on Neuromuscular and Mitochondrial Diseases, Vall d'Hebron Institut de Recerca, Universitat Autònoma de Barcelona, Barcelona, Spain. ${ }^{14}$ Departmento de Bioquímica y Biología Molecular, Universidad de Zaragoza, Instituto de Investigación Sanitaria de Aragón (IIS-Aragón), Zaragoza, Spain. ${ }^{15}$ Biochemistry and Molecular Biology Department, Institute of Biomedicine (IBUB), Institut de Recerca Sant Joan de Déu, University of Barcelona, Barcelona, Spain. ${ }^{16}$ Biomedical Network Research Centre on Obesity and Nutrition (CIBEROBN), Instituto de Salud Carlos III, Madrid, Spain. ${ }^{17}$ Clinical Biochemistry Department, Institut de Recerca Sant Joan de Déu, Hospital Sant Joan de Déu, Barcelona, Spain. ${ }^{18}$ Genetics Department, Faculty of Biology, University of Barcelona, Barcelona, Spain. ${ }^{\varpi}$ e-mail: cjimenezm@fsjd.org 
Mitochondrial DNA depletion and deletion syndrome 2 (MTDPS2) is caused by mutations in the nuclear gene TK2 that encodes thymidine kinase 2 (TK2) which is necessary for mitochondrial DNA replication and maintenance ${ }^{1}$. TK2 deficiency manifests predominantly as a myopathy and includes extremely severe and rapidly progressive early-onset forms with survival of less than two years, to milder forms with late or very late-onset, and a slower rate of progression, but with almost invariable respiratory involvement than shortens life expectancy ${ }^{2-4}$.

A new therapy for TK2 deficiency is under investigation, based on administration of oral deoxynucleosides. The rationale is to induce the synthesis of pyrimidine deoxynucleotides (dTTP and dCTP) via alternative enzyme pathways by means of supplementation with their precursors. Although some patients started with taking oral deoxynucleotides (dTMP and dCMP), after the demonstration in vitro and in vivo ${ }^{5,6}$ that deoxynucleosides are the active agents, the therapy was changed to deoxynucleosides (dThd and dCtd). The efficacy of oral deoxynucleoside treatment was demonstrated in pre-clinical studies ${ }^{7,8}$ and in a group of 16 patients treated under a compassionate use program ${ }^{9}$. Without any major side effect, the therapy had striking effects on early-onset severe myopathy patients, such as improvement in muscle strength, reduction or discontinuation of mechanical ventilation and gastrostomy feeding, and regaining the ability to walk. The patients also showed considerable functional improvements according to motor outcome measures in childhood-onset cases and at least stabilization in late-onset ones?.

Biomarkers are playing an increasingly important role in drug development and treatment implementation ${ }^{10}$. In addition to contributing to a better understanding of diseases, biomarkers provide more sensitive and specific means of diagnosing and ways to determine responses to new treatments. In this way, they help to streamline clinical trial efficacy and reduce uncertainty in regulatory decision-making, thus accelerating drug approval and drug access for patients.

According to BEST (The Biomarkers, EndpointS and other Tools, FDA-NIH Biomarker Working Group, https://www.ncbi.nlm.nih.gov/books/NBK326791/) a monitoring biomarker is a biomarker which is measured serially for assessing the status of a disease or for evidence of an effect of a medical product. A pharmacodynamics/response biomarker is used to show that a biological response has occurred in an individual who has been exposed to a medical product.

Growth Differentiation Factor 15 (GDF-15) is a cytokine that is induced in response to various stimuli and in different pathological situations where it is associated with disease progression and negative prognosis ${ }^{11-13}$. We previously demonstrated that GDF-15 is a valuable circulating diagnostic biomarker for mitochondrial diseases including mitochondrial DNA depletion and deletion syndromes, MELAS and KSS ${ }^{14-16}$. Fibroblast Growth Factor 21 (FGF-21) has also been reported to be elevated in a range of mitochondrial diseases, particularly in those with muscle involvement ${ }^{17}$. Determination of circulating GDF-15 and FGF-21 has greatly aided the diagnosis of mitochondrial pathologies since their sensitivity is significantly higher than other conventional biomarkers such as venous lactate level ${ }^{16,18}$.

The objective of this study was to explore the application of circulating GDF-15 as a biomarker of disease severity and prognosis as well as to monitor response to deoxynucleosides treatment in patients with TK2 deficiency, and compare it with FGF-21. Furthermore, since GDF-15 has been linked to inflammation in some diseases $^{13,19}$ we also investigated circulating levels of inflammatory cytokines.

\section{Results}

Patients. The 26 patients included span the spectrum of phenotypes associated with TK2 deficiency ${ }^{4}$. Pretreatment data are summarized in Table 1. Patients P25 and P26 had to stop treatment soon after initiation because of an increase in liver enzymes. They were not further analyzed. Follow-up data of P1 to P24 are available in Tables 2 and 3.

The 24 treated patients were grouped according to age at treatment initiation: group 1 (the paediatric group) started treatment before 16 years of age (P1 to P15), and group 2 (the adult group) started treatment after this age (P16 to P24). This grouping allowed us to compare GDF-15 and FGF-21 serum levels with aged-matched controls and takes into account the observation that age-related metabolic changes contribute to the efficacy of deoxynucleoside therapy ${ }^{8}$.

Group 1 consisted of 15 children (five female and ten male), six of them with early-onset and very rapid progression (P1, P3, P4, P5, P6, and P7) and nine with childhood-onset and an intermediate disease course (P2 and P8-P15). Group 2 consisted of nine patients (seven female and two male) and included one patient with childhood-onset (P22) and eight patients with late-onset forms (P16 to P21 and P23 to P24). To assess disease progression, patients were compared with other patients with similar clinical phenotypes based on previous publications $s^{2,4,9}$. The follow-up time ranged from 1 to 49 months for patients in group 1 and from 1 to 46 months for patients in group 2. A summary of the age of onset and treatment initiation for each group is provided in Table 4 .

Change of serum GDF-15 and FGF-21 over time in treated patients. We previously described the baseline levels of GDF-15 in a healthy pediatric population ${ }^{15}$. For the purposes of this study, we analyzed GDF-15 serum levels in 13 adult healthy controls (age range 23-56 years) and found that the mean GDF-15 concentration was $323 \mathrm{pg} / \mathrm{mL}$ (SE 19.7; range $232-460 \mathrm{pg} / \mathrm{mL}$ ) which is comparable to the mean values in the pediatric (age range 1 month to 18 years) control population $(350 \mathrm{pg} / \mathrm{mL}$; SE 20.7 , range $155-584)$ and overlaps with values reported elsewhere ${ }^{20}$. Baseline levels were available from 12 participants in group 1 and nine in group 2 . The mean values and ranges for GDF-15 and FGF-21 for both groups of patients are summarised in Table 4. GDF-15 levels were increased on average by 30 -fold in group 1 (mean $10716 \mathrm{pg} / \mathrm{mL}$; SE 2579; range 2641-27066), and by six-fold in group 2 (mean $1955 \mathrm{pg} / \mathrm{mL}$; SE 149; range 1261-2483), relative to the mean values in the control groups. If we only take into account the five patients from group 1 with the most severe disease form for whom we have basal measurements, the average GDF-15 levels were 60-fold increased over the value in the paediatric control group (mean 20246 pg/mL; SE 2371; range 14756-27066). 


\begin{tabular}{|c|c|c|c|c|c|c|c|c|c|c|c|c|c|}
\hline $\begin{array}{l}\text { CLINICAL } \\
\text { FORM }\end{array}$ & ID & SEX & $\begin{array}{l}\text { AGE AT } \\
\text { ONSET }\end{array}$ & BMI & $\begin{array}{l}\text { WALK } \\
\text { INDEPENDENTLY } \\
(\mathrm{Y} / \mathrm{N})\end{array}$ & $\begin{array}{l}\text { MECHANICAL } \\
\text { VENTILATION } \\
(\mathrm{Y} / \mathrm{N})\end{array}$ & $\begin{array}{l}\text { PEG } \\
(\mathrm{Y} / \mathrm{N})\end{array}$ & $\begin{array}{l}\text { GENOTYPE } \\
\text { (Allele 1/Allele 2) }\end{array}$ & $\begin{array}{l}\text { DEPLETION } \\
\text { (relative to } \\
\text { normal) }\end{array}$ & $\begin{array}{l}\text { MULTIPLE } \\
\text { DELETIONS } \\
(\mathrm{Y} / \mathrm{N})\end{array}$ & CK (UI/l) & $\begin{array}{l}\text { BASAL } \\
\text { GDF-15 } \\
(\mathrm{pg} / \mathrm{mL})\end{array}$ & $\begin{array}{l}\text { BASAL } \\
\text { FGF-21 } \\
(\mathrm{pg} / \mathrm{mL})\end{array}$ \\
\hline \multirow{15}{*}{$\begin{array}{l}\text { GROUP } \\
1 \text { (started } \\
\text { treatment } \\
<16)\end{array}$} & 1 & $\mathrm{~F}$ & $23 \mathrm{~m}$ & $<\mathrm{P} 3$ & $\mathrm{~N}$ & $\mathrm{~N}$ & $\mathrm{~N}$ & p.Lys202del/p.Asp177Tyr & $25 \%$ & $\mathrm{~N}$ & 1183 & 15.000 & 1373 \\
\hline & 2 & M & $30 \mathrm{~m}$ & $<\mathrm{P} 3$ & $\mathrm{~N}$ & $\mathrm{Y}$ & NGT & p.His121Asn/p.His12Asn & $50 \%$ & $\mathrm{Y}$ & 538 & 4608 & 402 \\
\hline & 3 & M & $17 \mathrm{~m}$ & P3 & $\mathrm{N}$ & $\mathrm{Y}$ & NGT & p.Tyr208Cys/p.Arg130Trp & $15 \%$ & $\mathrm{~N}$ & 148 & 14756 & 3013 \\
\hline & 4 & M & $15 \mathrm{~m}$ & $<\mathrm{P} 3$ & $\mathrm{~N}$ & $\mathrm{Y}$ & $\mathrm{N}$ & p.His121Asn/p.Arg192Lys & $13 \%$ & $\mathrm{~N}$ & 284 & ND & $\mathrm{ND}$ \\
\hline & 5 & M & $13 \mathrm{~m}$ & $<\mathrm{P} 3$ & $\mathrm{~N}$ & $\mathrm{Y}$ & NGT & p.Thr108Met/Leu215Pro & ND & ND & 872 & 21641 & 4088 \\
\hline & 6 & M & $13 \mathrm{~m}$ & $<\mathrm{P} 3$ & $\mathrm{~N}$ & $\mathrm{Y}$ & NGT & p.Thr108Met/Leu215Pro & ND & ND & 949 & 22767 & 3244 \\
\hline & 7 & M & $13 \mathrm{~m}$ & $<\mathrm{P} 3$ & $\mathrm{~N}$ & $\mathrm{~N}$ & $\mathrm{~N}$ & p.Lys85*/p.Pro154Leu & ND & ND & 921 & 27066 & 7531 \\
\hline & 8 & M & $24 \mathrm{~m}$ & $\mathrm{ND}$ & ND & $\mathrm{ND}$ & $\mathrm{ND}$ & p.Thr108Met/p.Thr108Met & $\mathrm{ND}$ & ND & 285 & 4597 & ND \\
\hline & 9 & M & $31 \mathrm{~m}$ & ND & $\mathrm{ND}$ & ND & ND & p.Thr108Met/p.Thr108Met & $8 \%$ & ND & 626 & 6825 & ND \\
\hline & 10 & $\mathrm{~F}$ & $3 y$ & P3 & $\mathrm{Y}$ & $\mathrm{Y}$ & NGT & p.Asn58Ser/p.Asn58Ser & ND & ND & 1789 & 4797 & 809 \\
\hline & 11 & M & $3 y$ & P50 & $\mathrm{Y}$ & $\mathrm{N}$ & $\mathrm{N}$ & p.Asn58Ser/p.Asn58Ser & ND & $\mathrm{ND}$ & 1036 & 2641 & 398 \\
\hline & 12 & $\mathrm{~F}$ & $2 y$ & P50 & $\mathrm{Y}$ & $\mathrm{N}$ & $\mathrm{N}$ & p.His121Asn/p.Arg183Trp & ND & ND & 460 & 3686 & 786 \\
\hline & 13 & $\mathrm{~F}$ & $2 y$ & P50 & $\mathrm{Y}$ & $\mathrm{N}$ & $\mathrm{N}$ & p.His121Asn/p.Arg183Trp & ND & ND & 945 & 3841 & 471 \\
\hline & 14 & M & $3 y$ & P3 & $\mathrm{N}$ & $\mathrm{Y}$ & $\mathrm{N}$ & p.His121Asn/p.Arg183Trp & ND & ND & 184 & 5006 & 1057 \\
\hline & 15 & $\mathrm{~F}$ & $6 y$ & $<\mathrm{P} 3$ & $\mathrm{Y}$ & $\mathrm{N}$ & $\mathrm{N}$ & p.Asn58Ser/p.Asn58Ser & ND & ND & 742 & 2781 & 595 \\
\hline \multirow{11}{*}{$\begin{array}{l}\text { GROUP } \\
2 \text { (started } \\
\text { treatment } \\
>16)\end{array}$} & 16 & $\mathrm{~F}$ & $>12 y$ & 13.86 & $\mathrm{Y}$ & $\mathrm{Y}$ & $\mathrm{N}$ & p.Thr108Met/p.Thr108Met & $17 \%$ & $\mathrm{Y}$ & 2435 & 2423 & 666 \\
\hline & 17 & M & $50 y$ & 27.7 & $\mathrm{Y}$ & $\mathrm{Y}$ & $\mathrm{N}$ & p.Lys202del/p.Lys202del & $66 \%$ & $\mathrm{Y}$ & 357 & 1529 & 252 \\
\hline & 18 & $\mathrm{~F}$ & $30 \mathrm{y}$ & 27.12 & $\mathrm{Y}$ & $\mathrm{Y}$ & $\mathrm{N}$ & p.Lys202del/p.Lys202del & $60 \%$ & $\mathrm{Y}$ & 294 & 1695 & 353 \\
\hline & 19 & $\mathrm{~F}$ & $20 y$ & 17.79 & $\mathrm{Y}$ & $\mathrm{Y}$ & $\mathrm{Y}$ & p.Thr108Met/p.Thr108Met & $39 \%$ & $\mathrm{Y}$ & 303 & 2439 & 197 \\
\hline & 20 & $\mathrm{~F}$ & $60 y$ & 26.01 & $\mathrm{Y}$ & $\mathrm{Y}$ & $\mathrm{N}$ & p.Lys202del/p.Lys202del & $\mathrm{ND}$ & ND & 647 & 2483 & 897 \\
\hline & 21 & M & $30 \mathrm{y}$ & 23.7 & $\mathrm{Y}$ & $\mathrm{N}$ & $\mathrm{N}$ & p.Lys202del/p.Lys202del & $\mathrm{ND}$ & $\mathrm{Y}$ & 350 & 1640 & 185 \\
\hline & 22 & $\mathrm{~F}$ & $5 y$ & 26.17 & $\mathrm{Y}$ & $\mathrm{Y}$ & $\mathrm{N}$ & p.Thr108Met/p.Thr108Met & ND & $\mathrm{Y}$ & 548 & 2149 & 943 \\
\hline & 23 & $\mathrm{~F}$ & $30 y$ & ND & $\mathrm{Y}$ & $\mathrm{Y}$ & $\mathrm{N}$ & p.Thr108Met/p.Thr108Met & $53 \%$ & $\mathrm{Y}$ & 233 & 1261 & 190 \\
\hline & 24 & $\mathrm{~F}$ & $20 y$ & 20,5 & $\mathrm{Y}$ & $\mathrm{Y}$ & GT & p.Lys202del/p.Ala420Val & ND & ND & 1142 & 1979 & 179 \\
\hline & 25 & $\mathrm{~F}$ & $50 \mathrm{Y}$ & 28,7 & $\mathrm{Y}$ & $\mathrm{Y}$ & $\mathrm{N}$ & p.Lys202del/p.Lys202del & ND & $\mathrm{Y}$ & 405 & ND & ND \\
\hline & 26 & $\mathrm{~F}$ & $14 \mathrm{Y}$ & 24,5 & $\mathrm{Y}$ & $\mathrm{Y}$ & $\mathrm{N}$ & p.Thr108Met/p.Thr108Met & $19 \%$ & $\mathrm{Y}$ & 425 & ND & ND \\
\hline
\end{tabular}

Table 1. Clinical, biochemical and molecular characteristics of patients, before treatment. BMI, body-mass index; CK, creatine kinase; F, female; GDF-15, differentiation growth factor 15; M, male; m, months; ND, not determined; NGT, nasogastric tube; N, No; P, percentile; PEG, percutaneous endoscopic gastrostomy; $y$, years; Y, yes.

For FGF-21, average baseline levels were elevated by 25 -fold in group 1 (mean 1981 pg/mL; SE 702; range 402731 ) and by six-fold in group 2 (mean $429 \mathrm{pg} / \mathrm{mL}$; SE 106; range 179-943), relative to control values ${ }^{16}$. Similarly to GDF-15, the average basal FGF-21 value almost doubled if we only took into account the most severe patients from group 1 (Table 4).

Thus, both GDF-15 and FGF-21 basal serum levels are associated with the severity of the phenotype. This was, however, not the case for $\mathrm{CK}$ since its basal levels were comparable regardless of the severity of the phenotype (Fig. 1).

The development of serum GDF-15 values at different times after the initiation of treatment is represented in Fig. 1. We applied a linear mixed model using the log-transformed GDF-15 concentration levels and found a significant decrease over time in patients in both group $1(\mathrm{p}<0.0001)$ and group $2(\mathrm{p}=0.0082)$ (Fig. 2A,B). FGF-21 followed a similar trend, although not significant, for group $1(\mathrm{p}=0.062)$ but this trend was not present in group 2 (Fig. 2C,D).

Because the time points at which GDF-15 levels were measured are not uniform across patients and to homogenize the data we grouped the measurements at regular intervals corresponding to the follow-up intervals for each group (Supp. Fig. 1). We observed that in group 1 GDF-15 values decreased steadily over time, particularly between 0 and 24 months and that the difference between intervals was statistically significant (Kruskal-Wallis chi-squared $\mathrm{p}<0.001)$. After 12 months of treatment, most values were very close or below the normal threshold $(550 \mathrm{pg} / \mathrm{mL})$ with the exception of $\mathrm{P} 2$.

In patient P2 the GDF-15 concentration already decreased to almost normal levels after only four months of treatment. For this patient the next measurement was performed after two years of treatment and a few days after the nucleoside treatment dose was reduced to $300 \mathrm{mg} / \mathrm{Kg}$ (and remained at this dose) because of diarrhea, and at this point GDF-15 was increased (to half the baseline level). Three months later, GDF-15 had almost returned to normal levels in P2. We do not know when exactly the increase in GDF-15 occurred and whether it happened before or during the few days interval between the change of dose and the measurement.

When we analysed FGF-21 levels, we found significant differences between the intervals $(\mathrm{p}=0.01)$ although after 12 months of treatment half of the patients still had levels above the normal threshold (Supp. Fig. 1). 


\begin{tabular}{|c|c|c|c|c|c|c|c|c|c|c|c|c|c|c|c|c|}
\hline \multirow{5}{*}{\multicolumn{2}{|c|}{\begin{tabular}{|l|l} 
ID & \\
AGE OF ONSET \\
AGE AT INITIATION OF \\
TREATMENT \\
CURRENT AGE \\
TREATMENT DOSE $(\mathrm{mg} / \mathrm{kg} / \mathrm{d})$
\end{tabular}}} & 1 & 2 & 3 & 4 & 5 & 6 & 7 & 8 & 9 & 10 & 11 & 12 & 13 & 14 & 15 \\
\hline & & $23 \mathrm{~m}$ & $30 \mathrm{~m}$ & $17 \mathrm{~m}$ & $15 \mathrm{~m}$ & $13 \mathrm{~m}$ & $13 \mathrm{~m}$ & $13 \mathrm{~m}$ & $24 \mathrm{~m}$ & $31 \mathrm{~m}$ & $36 \mathrm{~m}$ & $36 \mathrm{~m}$ & $24 \mathrm{~m}$ & $24 \mathrm{~m}$ & $36 \mathrm{~m}$ & $6 y$ \\
\hline & & $27 \mathrm{~m}$ & $10 y$ & $30 \mathrm{~m}$ & $2.5 \mathrm{y}$ & $15 \mathrm{~m}$ & $15 \mathrm{~m}$ & $14 \mathrm{~m}$ & $33 \mathrm{~m}$ & $6 y$ & $14 \mathrm{y}$ & $12 y$ & $3 y$ & $3 y$ & $7 y$ & $9 y$ \\
\hline & & $5 y$ & $13 y$ & $7 y$ & $7 y$ & $21 \mathrm{~m}$ & $21 \mathrm{~m}$ & $24 \mathrm{~m}$ & $3 y$ & $7 y$ & $16 \mathrm{y}$ & $13 y$ & $5 y$ & $5 y$ & $9 y$ & $10 y$ \\
\hline & & 400 & 300 & 400 & 400 & 400 & 400 & 400 & 400 & 400 & 400 & 400 & 400 & 400 & 400 & 400 \\
\hline \multirow{5}{*}{ CK } & BASAL & 1183 & 538 & 148 & 284 & 872 & 940 & 921 & 285 & 626 & 1789 & 759 & 460 & 945 & 184 & 742 \\
\hline & 0-6 Months & ND & 316 & ND & ND & 649 & 561 & 275 & 133 & 175 & 235 & 243 & 606 & 857 & 164 & 269 \\
\hline & 6-12 Months & 114 & ND & 68 & 132 & ND & ND & 164 & 130 & 165 & 273 & 290 & 173 & 87 & 111 & 195 \\
\hline & 12-24 Months & 55 & 712 & ND & 116 & ND & ND & ND & ND & ND & ND & ND & ND & ND & ND & ND \\
\hline & $>24$ Months & 87 & 285 & ND & 61 & ND & ND & ND & ND & ND & ND & ND & ND & ND & $\mathrm{ND}$ & ND \\
\hline \multirow{5}{*}{ GDF-15 (pg/mL) } & BASAL & 15000 & 4608 & 14756 & ND & 21641 & 22767 & 27066 & ND & ND & 4797 & 2641 & 3686 & 3841 & 5006 & 2781 \\
\hline & 0-6 Months & 1200 & 762 & ND & ND & 6628 & 2174 & 588 & 4597 & 6825 & 4753 & 3105 & ND & 2635 & 5180 & 2249 \\
\hline & 6-12 Months & 497 & ND & 5093 & 2021 & ND & ND & ND & 396 & 369 & ND & ND & 341 & 330 & 440 & 345 \\
\hline & 12-24 Months & 392 & 2084 & 594 & 255 & ND & ND & ND & ND & ND & ND & ND & ND & ND & ND & ND \\
\hline & $>24$ Months & 288 & 631 & ND & 284 & ND & ND & ND & ND & ND & ND & ND & ND & ND & ND & ND \\
\hline \multirow{5}{*}{ FGF-21 (pg/mL) } & BASAL & 1373 & 402 & 3013 & ND & 4088 & 3245 & 7531 & ND & ND & 809 & 398 & 786 & 471 & 1057 & 595 \\
\hline & 0-6 Months & 72 & 228 & ND & ND & 1445 & 776 & 208 & 583 & 743 & 769 & 1090 & ND & 351 & 1077 & 417 \\
\hline & 6-12 Months & 31 & ND & 2170 & 415 & ND & ND & ND & ND & ND & ND & ND & 42 & $<$ LOQ & $<$ LOQ & 34 \\
\hline & 12-24 Months & 30 & 689 & 603 & 51 & ND & ND & ND & ND & ND & ND & ND & ND & ND & ND & ND \\
\hline & $>24$ Months & 37 & 134 & ND & 100 & ND & ND & ND & ND & ND & ND & ND & ND & ND & ND & ND \\
\hline \multirow{5}{*}{ BMI } & BASAL & $<\mathrm{P} 3$ & $<\mathrm{P} 3$ & P3 & P1 & 13,2 & 13,5 & 12,7 & ND & ND & 15,3 & 20,2 & 13,5 & 16 & 11,5 & 10,5 \\
\hline & 0-6 Months & ND & 9.6 & ND & ND & 12,8 & 13,2 & 12,9 & ND & ND & 16,1 & 19,05 & 15,02 & 15,08 & 11 & 10,7 \\
\hline & 6-12 Months & 17.2 & ND & 16.02 & 12.75 & ND & ND & ND & ND & ND & $\mathrm{ND}$ & ND & ND & ND & ND & ND \\
\hline & 12-24 Months & 16.3 & 20 & ND & ND & ND & ND & ND & ND & ND & ND & ND & ND & ND & ND & ND \\
\hline & $>24$ Months & 14.9 & 25.3 & ND & ND & ND & ND & ND & ND & ND & ND & ND & ND & ND & ND & ND \\
\hline \multirow{5}{*}{$6 \mathrm{MWT}$} & BASAL & 0 & ND & ND & ND & ND & ND & ND & ND & 420 & 75 & 342 & 250 & 272 & 0 & 307 \\
\hline & 0-6 Months & 150 & ND & ND & ND & ND & ND & ND & ND & 531 & 350 & 354 & ND & ND & ND & 364 \\
\hline & 6-12 Months & 250 & ND & ND & ND & ND & ND & ND & ND & 512 & 379 & 375 & 394 & 368 & 15 & ND \\
\hline & 12-24 Months & 391 & ND & ND & ND & ND & ND & ND & ND & ND & ND & ND & ND & ND & ND & ND \\
\hline & $>24$ Months & 477 & ND & ND & ND & ND & ND & ND & ND & ND & ND & ND & ND & ND & ND & ND \\
\hline \multirow{5}{*}{ EK2 } & BASAL & ND & 26 & 30 & 28 & ND & ND & ND & ND & ND & ND & ND & ND & ND & ND & ND \\
\hline & 0-6 Months & ND & ND & ND & ND & ND & ND & ND & ND & ND & ND & ND & ND & $\mathrm{ND}$ & ND & ND \\
\hline & 6-12 Months & ND & 9 & 21 & 14 & ND & ND & ND & ND & ND & ND & ND & ND & ND & ND & ND \\
\hline & 12-24 Months & ND & ND & ND & 12 & ND & ND & ND & ND & ND & ND & ND & ND & ND & ND & ND \\
\hline & $>24$ Months & ND & 13 & ND & 11 & ND & ND & ND & ND & ND & ND & ND & ND & ND & ND & ND \\
\hline \multirow{5}{*}{ NSAA } & BASAL & 4 & ND & ND & ND & ND & ND & ND & 20 & 27 & ND & ND & ND & ND & ND & ND \\
\hline & 0-6 Months & 6 & ND & ND & ND & ND & ND & ND & ND & 33 & ND & ND & ND & ND & ND & ND \\
\hline & 6-12 Months & 20 & ND & ND & ND & ND & $\mathrm{ND}$ & ND & 31 & 33 & ND & ND & ND & ND & ND & ND \\
\hline & 12-24 Months & 26 & ND & ND & ND & ND & ND & ND & ND & ND & ND & ND & ND & ND & ND & ND \\
\hline & $>24$ Months & ND & ND & ND & ND & ND & ND & ND & ND & ND & ND & ND & ND & ND & ND & ND \\
\hline \multirow{5}{*}{ HMFS } & BASAL & ND & ND & ND & ND & ND & ND & ND & ND & ND & 21 & 35 & 38 & 38 & 17 & 38 \\
\hline & 0-6 Months & ND & ND & ND & ND & ND & ND & ND & ND & ND & 30 & 33 & ND & ND & ND & 39 \\
\hline & 6-12 Months & ND & ND & ND & ND & ND & ND & ND & ND & ND & 36 & 37 & 39 & 39 & 23 & ND \\
\hline & 12-24 Months & ND & ND & ND & ND & ND & $\mathrm{ND}$ & ND & ND & ND & $\mathrm{ND}$ & ND & ND & ND & ND & ND \\
\hline & $>24$ Months & ND & ND & ND & ND & ND & ND & ND & ND & ND & ND & ND & ND & ND & ND & ND \\
\hline \multirow{5}{*}{ HMFSE } & BASAL & ND & ND & ND & ND & ND & ND & ND & ND & ND & 26 & 51 & 51 & 53 & 19 & 62 \\
\hline & 0-6 Months & ND & ND & ND & ND & ND & ND & ND & ND & ND & 48 & 49 & ND & ND & ND & 65 \\
\hline & 6-12 Months & ND & ND & ND & ND & ND & $\mathrm{ND}$ & ND & ND & ND & 57 & 55 & 65 & 65 & 27 & ND \\
\hline & 12-24 Months & ND & ND & ND & ND & ND & ND & ND & ND & ND & ND & ND & ND & ND & ND & ND \\
\hline & $>24$ Months & ND & ND & ND & ND & ND & ND & ND & ND & ND & ND & ND & ND & ND & ND & ND \\
\hline \multirow{5}{*}{ CHOP INTEND } & BASAL & ND & ND & ND & ND & 42 & 45 & 17 & ND & ND & ND & ND & ND & ND & ND & ND \\
\hline & 0-6 Months & ND & ND & ND & ND & 45 & 54 & 64 & ND & ND & ND & ND & ND & ND & ND & ND \\
\hline & 6-12 Months & ND & ND & ND & ND & ND & ND & ND & ND & ND & ND & ND & ND & ND & ND & ND \\
\hline & 12-24 Months & ND & ND & ND & ND & ND & ND & ND & ND & ND & ND & ND & ND & $\mathrm{ND}$ & $\mathrm{ND}$ & ND \\
\hline & $>24$ Months & ND & ND & ND & ND & ND & ND & ND & ND & ND & ND & ND & ND & ND & ND & ND \\
\hline
\end{tabular}




\begin{tabular}{|c|c|c|c|c|c|c|c|c|c|c|c|c|c|c|c|c|}
\hline ID & & 1 & 2 & 3 & 4 & 5 & 6 & 7 & 8 & 9 & 10 & 11 & 12 & 13 & 14 & 15 \\
\hline \multirow{5}{*}{ FVC (\%) } & BASAL & ND & 13 & ND & ND & ND & ND & ND & ND & ND & ND & ND & ND & ND & ND & 38 \\
\hline & 0-6 Months & ND & ND & ND & ND & ND & ND & ND & ND & ND & 19 & 50 & ND & ND & ND & 66 \\
\hline & 6-12 Months & ND & 22 & ND & ND & ND & ND & ND & ND & ND & 35 & 70 & ND & ND & 71 & ND \\
\hline & 12-24 Months & ND & ND & ND & ND & ND & ND & ND & ND & ND & ND & ND & ND & ND & ND & ND \\
\hline & $>24$ Months & ND & 26 & ND & ND & ND & ND & ND & ND & ND & ND & ND & ND & ND & ND & ND \\
\hline
\end{tabular}

Table 2. Outcome measures, Group 1 (age at treatment initiation <16 years). CK, creatine kinase; EK, Egen Klassifikation; HMFS, Hammersmith Motor Functional Scale; HMFSE, Hammersmith Motor Functional Scale Expanded; 6MWT, 6-minute walk test; m, months; ND, not determined; NSAA, North Star Ambulatory Assessment; $y$, years.

In the group that started treatment at an older age ( $>16$ years-old), consisting mainly of patients with a late-onset phenotype, biomarker determinations spanned a longer period of time ( 0 to $>36$ months). Although the differences in GDF-15 levels between the different intervals were significant (Kruskal-Wallis chi-squared $\mathrm{p}=0.003$ ), levels appeared to decrease mainly during the first 12 months of treatment and then remained stable (Supp. Fig. 1). In contrast, there was no clear trend for FGF-21 and in some patients levels even increased with treatment. In this case, the difference between intervals was not significant (Supp. Fig. 1).

We calculated the rate of decline of GDF-15 and FGF-21 levels relative to the basal levels in the same time intervals in the paediatric and adult patients for whom we had measurements before treatment (\% change at time $\mathrm{T}=\log$ GDF-15 at T-log GDF-15 at T0/log GDF-15 T0 x 100). The rate of decline increased with time in group 1 for GDF-15 (-12.5\% between 0 and 6 months, $-26.7 \%$ between 6 and 12 months, $-26.9 \%$ between 12 and 24 months and $32.3 \%$ after 24 months) and FGF- $21(-11.5 \%,-36.3 \%,-21.3 \%$ and $-34.2 \%$ in the same intervals) whereas it was rather uniform in group 2 for GDF-15 (-10.3\% between 0 and 6 months, $-12.8 \%$ between 6 and 12 months, $-12.5 \%$ between 12 and 36 months, and $-12.6 \%$ after 36 months) and for FGF- 21 ( $-15.4 \%,-8.4 \%$, $-14 \%$ and $-9.1 \%$ for the same time intervals).

Change of serum GDF-15 over time in patients that stopped treatment. Patients P25 and P26 stopped treatment because their liver enzymes had increased. P25 received treatment for three months, then stopped for three months, and then took nucleosides for one more month before stopping treatment completely. We do not have baseline levels for P25. The first GDF-15 measurement, performed after 6 months without treatment, was elevated ( $1833 \mathrm{pg} / \mathrm{mL})$. One month later, GDF-15 levels had decreased to $1489 \mathrm{pg} / \mathrm{mL}$, two months later ( 9 months after stopping the treatment completely) GDF-15 went up to $1675 \mathrm{pg} / \mathrm{mL}$. Then they remained elevated $(1657 \mathrm{pg} / \mathrm{mL})$ even after 18 months of stopping the treatment when the last measurement was taken.

P26 received treatment for three months, stopped for four months, and then restarted treatment for one more month before stopping completely. GDF-15 levels were measured nearly three months after treatment interruption and were then mildly elevated $(688 \mathrm{pg} / \mathrm{ml})$. Then, in this patient, they remained stable over the next four months (including one month with treatment) to $639 \mathrm{pg} / \mathrm{mL}$. Three months later $(10$ months after the first treatment interruption) it had risen to $818 \mathrm{pg} / \mathrm{ml}$. From then onwards, levels continued to rise ( $1301 \mathrm{pg} / \mathrm{mL}$ after 13 months of treatment interruption) to reach $1656 \mathrm{pg} / \mathrm{mL}$ at the last measurement, 16 months after treatment interruption.

Thus, the trend of GDF-15 in the absence of treatment appears to reflect the disease course in these two patients, P25 has remained clinically stable whilst P26 has a more progressive form of the disease.

The comparison between GDF-15 concentrations in treated adult patients and these two patients that stopped over a similar time frame ( 0 to 28 months) is shown in Fig. 3. While in the treated patients GDF-15 levels declined, in the two patients that stopped they went up or remained stable but did not lower in either case.

Clinical outcome measures and correlation analysis. We analyzed several clinical outcomes in both patients groups to determine whether the decline in GDF-15 was associated with clinical improvement following treatment. Tables 2 and 3 and Fig. 4 summarise this information.

Patients P1, P3, and P4 are the patients P5, P2, and P1 in our previous report ${ }^{9}$. They are in the early-onset and severe myopathy group in which the response to treatment was striking. This group of patients was defined by (1) onset before 24 months and (2) inability to walk, use of mechanical ventilation, or both within one year of onset. Without treatment, only $27.3 \%$ of patients described in the literature with such characteristics survived at least two years after onset $(95 \% \mathrm{CI}=0.17-0.45)^{9}$. In contrast, with treatment, $\mathrm{P} 1, \mathrm{P} 3$, and $\mathrm{P} 4$ are still alive, four, six, and six years after disease onset respectively. One of these patients was weaned of ventilatory support and the two others regained the ability to walk without assistance. Patients P5, P6, and P7, not reported previously, also represent the most severe phenotype with early-onset and very rapid progression course. After treatment, they were evaluated with the Children's Hospital of Philadelphia Infant Test of Neuromuscular Disorders (CHOP INTEND) assessments, which showed a mean improvement of 13.7 points after only three months of follow-up, and one (P7) even reached the maximum score on the scale three months later, which indicates complete motor recovery after only six months of treatment.

In childhood-onset cases, when we compared the latest post-treatment value (around one year after the start of the treatment) to the pre-treatment value for the more frequent outcome measures used, we found a significant improvement in the distance walked on the 6-minutes walking test (6MWT), with a mean increase of 105.8 meters $(\mathrm{p}=0.01)$ (Fig. 4A). We also found a significant improvement in the scores of the Hammersmith Motor Function 


\begin{tabular}{|c|c|c|c|c|c|c|c|c|c|c|}
\hline \multicolumn{2}{|l|}{ ID } & 16 & 17 & 18 & 19 & 20 & 21 & 22 & 23 & 24 \\
\hline \multirow{4}{*}{\multicolumn{2}{|c|}{$\begin{array}{l}\text { AGE OF ONSET } \\
\text { AGE AT INITIATION OF } \\
\text { TREATMENT } \\
\text { CURRENT AGE } \\
\text { TREATMENT DOSE }(\mathrm{mg} / \mathrm{kg} / \mathrm{d})\end{array}$}} & $>12 y$ & $50 y$ & $30 \mathrm{y}$ & $20 y$ & $60 y$ & $36 y$ & $5 y$ & $30 y$ & $20 y$ \\
\hline & & $30 \mathrm{y}$ & $58 \mathrm{y}$ & $59 y$ & $31 y$ & $74 y$ & $60 y$ & $35 y$ & $46 y$ & $57 y$ \\
\hline & & $34 \mathrm{y}$ & $62 y$ & $62 y$ & $34 y$ & $75 y$ & $60 y$ & $36 y$ & $46 y$ & $60 y$ \\
\hline & & 320 & 300 & 400 & 400 & 400 & 400 & 350 & 300 & 400 \\
\hline \multirow{5}{*}{ CK (UI/l) } & BASAL & 2435 & 525 & 294 & 303 & 647 & 402 & 548 & 188 & 1142 \\
\hline & 0-6 Months & ND & ND & 123 & 322 & ND & 264 & 107 & ND & 1195 \\
\hline & 6-12 Months & 393 & ND & 258 & 292 & 105 & ND & 142 & ND & 1348 \\
\hline & 12-36 Months & 330 & 351 & 108 & 110 & ND & ND & ND & ND & ND \\
\hline & >36 Months & 561 & 459 & 185 & 467 & 89 & ND & ND & ND & ND \\
\hline \multirow{5}{*}{ GDF-15 (pg/mL) } & BASAL & 2423 & 1529 & 1695 & 2439 & 2483 & 1640 & 2149 & 1261 & 1980 \\
\hline & 0-6 Months & ND & ND & ND & ND & 1364 & 1357 & 473 & 486 & 1052 \\
\hline & 6-12 Months & ND & ND & ND & ND & 1320 & ND & 413 & ND & 1011 \\
\hline & 12-36 Months & 464 & 782 & 704 & 1110 & 1099 & ND & ND & ND & ND \\
\hline & $>36$ Months & 480 & 1137 & 753 & 783 & ND & ND & ND & ND & ND \\
\hline \multirow{5}{*}{ FGF-21 (pg/mL) } & BASAL & 666 & 252 & 353 & 197 & 897 & 185 & 943 & 190 & 179 \\
\hline & 0-6 Months & ND & ND & ND & ND & 732 & 135 & 47 & ND & 113 \\
\hline & 6-12 Months & ND & ND & ND & ND & 344 & ND & ND & ND & 155 \\
\hline & 12-36 Months & 1222 & 944 & 245 & 915 & ND & ND & ND & ND & ND \\
\hline & $>36$ Months & 632 & 1025 & 421 & ND & ND & ND & ND & ND & ND \\
\hline \multirow{5}{*}{ BMI } & BASAL & 13.86 & 27.7 & 27.12 & 17.79 & 26.01 & 23.7 & 26.17 & ND & 20,8 \\
\hline & 0-6 Months & ND & ND & ND & 19,06 & 25 & 23.6 & 25.4 & ND & 21 \\
\hline & 6-12 Months & ND & ND & ND & 21,09 & 27,6 & ND & ND & ND & 20,4 \\
\hline & 12-36 Months & 16.06 & ND & 26.3 & 21.23 & ND & ND & ND & ND & ND \\
\hline & $>36$ Months & ND & ND & 26.9 & 18,63 & ND & ND & ND & ND & ND \\
\hline \multirow{5}{*}{$6 \mathrm{MWT}$} & BASAL & 532 & 475 & 386 & 390 & 345 & ND & 368 & 413 & 225 \\
\hline & 0-6 Months & ND & 500 & 355 & 437 & ND & ND & 435 & 515 & 251 \\
\hline & 6-12 Months & ND & ND & 400 & 474 & ND & ND & 450 & ND & 228 \\
\hline & 12-36 Months & 550 & 435 & 354 & 369 & ND & ND & ND & ND & ND \\
\hline & $>36$ Months & 600 & 442 & 450 & 476 & ND & ND & ND & ND & ND \\
\hline \multirow{5}{*}{ NSAA } & BASAL & ND & ND & 26 & 21 & 29 & ND & 16 & 30 & ND \\
\hline & 0-6 Months & 30 & 24 & 26 & 21 & ND & ND & 25 & 31 & ND \\
\hline & 6-12 Months & ND & ND & 29 & 23 & ND & ND & 26 & ND & ND \\
\hline & 12-36 Months & 29 & 27 & 32 & 28 & ND & ND & ND & ND & ND \\
\hline & $>36$ Months & 30 & ND & 31 & 29 & ND & ND & ND & ND & ND \\
\hline \multirow{5}{*}{ PIM (\%) } & BASAL & 25 & 40 & 49.9 & 5 & 59 & ND & 37 & 31 & ND \\
\hline & 0-6 Months & ND & ND & 40.2 & ND & 33,9 & ND & 37,8 & ND & ND \\
\hline & 6-12 Months & 29.5 & ND & 53 & 24 & 47.3 & ND & ND & ND & ND \\
\hline & 12-36 Months & 21.4 & 38.6 & 49.9 & ND & 39,8 & ND & ND & ND & ND \\
\hline & >36 Months & ND & 28 & 53 & 27 & ND & ND & ND & ND & ND \\
\hline \multirow{5}{*}{ FVC (\%) } & BASAL & 46.5 & 45 & 71.8 & 26 & 69 & 78 & 53.2 & 63.7 & ND \\
\hline & 0-6 Months & ND & 51.4 & 79.5 & 33 & 66,5 & 82.6 & 62 & 64 & 16 \\
\hline & 6-12 Months & 50.3 & ND & 75 & 23,7 & 78,9 & ND & ND & ND & 17 \\
\hline & 12-36 Months & 46.7 & 46.7 & 77.7 & 32,2 & 83,4 & ND & ND & ND & ND \\
\hline & $>36$ Months & 50.5 & 43.6 & 72 & 34,8 & ND & ND & ND & $\mathrm{ND}$ & ND \\
\hline
\end{tabular}

Table 3. Outcome measures, Group 2 (age at treatment initiation $>16$ years). ND, not determined; CK, creatine kinase; $y$, years; m, months; NA, not available, GDF-15, growth differentiation factor 15; FGF-21, fibroblast growth factor 21; BMI, body mass index; 6MWT, six-minute walk test; NSAA, north star ambulatory assessment; PIM, maximal inspiratory pressure; FVC, forced vital capacity.

Scale (HMFS) and the Hammersmith Motor Function Scale-Expanded HMFSE scales (Fig. 4B,C), with an average improvement of 4.3 points and 12 points respectively between the pre-treatment and last post-treatment determinations ( $\mathrm{p}=0.03$ in both cases) (Fig. 4A). Other clinical outcome measures (Egen Klassifikation Scale 2, EK2; North Star Ambulatory Assessment, NSAA) also reflected improvements, although without reaching statistical significance, when pre-treatment and post-treatment scores were compared. We did not have enough basal data regarding respiratory function (Forced Vital Capacity, FVC) to perform specific analyses in this group of patients (Fig. 4D). 


\begin{tabular}{|l|l|l|l|}
\hline Group 1 & Mean & SE & Range \\
\hline Age onset & $27.1 \mathrm{~m}$ & 3.8 & $13-72 \mathrm{~m}$ \\
\hline Age treatment start & $62.1 \mathrm{~m}$ & 12.6 & $14-168 \mathrm{~m}$ \\
\hline Basal GDF-15 $(\mathrm{pg} / \mathrm{mL})$ & 10716 & 2579 & $2641-27066$ \\
\hline Basal FGF-21 (pg/mL) & 1981 & 702 & $402-7531$ \\
\hline Group 2 & $29.2 \mathrm{y}$ & 5.3 & $5-60 \mathrm{y}$ \\
\hline Age onset & $50 \mathrm{y}$ & 4.3 & $30-74 \mathrm{y}$ \\
\hline Age treatment start & 1955 & 149 & $1261-2483$ \\
\hline Basal GDF-15 $(\mathrm{pg} / \mathrm{mL})$ & 429 & 106 & $179-943$ \\
\hline Basal FGF-21 $(\mathrm{pg} / \mathrm{mL})$ & \multicolumn{5}{|l}{} \\
\hline
\end{tabular}

Table 4. Summary of descriptive statistics. m, months; $y$, years; SE, standard error of the mean.

GDF-15

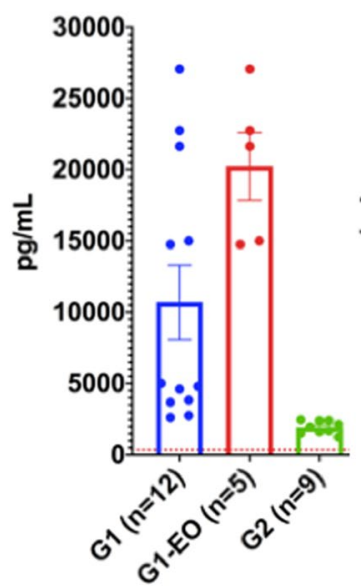

FGF-21

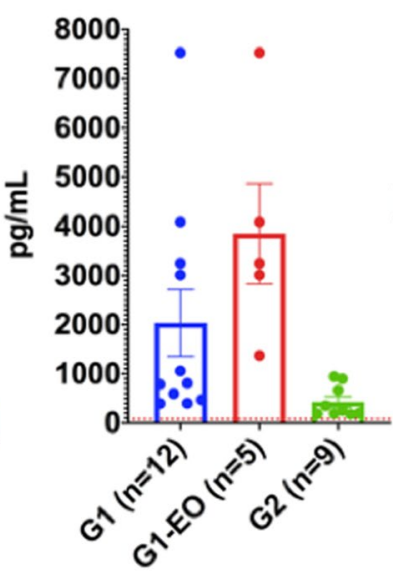

CK

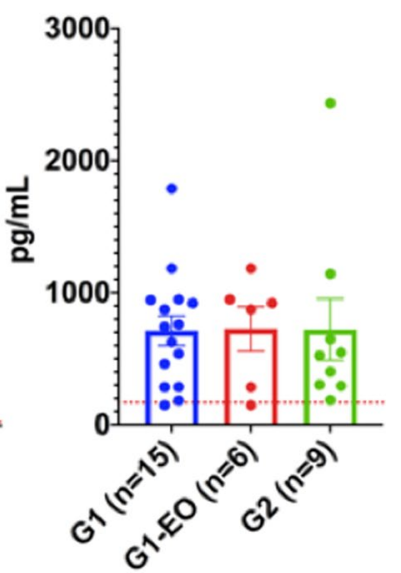

Figure 1. Pre-treatment GDF-15 and FGF-21 serum levels are associated with disease severity. Histograms representing the basal (before treatment) serum levels (mean and SEM) of GDF-15, FGF-21 and creatine kinase (CK) in patients from Group 1 (G1), those patients from Group 1 with the early onset and the most severe phenotype (G1-EO) and patients in Group 2 (G2). Dotted lines represent control values.

In the adult group, consisting mainly of patients with late-onset disease, the 6MWT and the FVC showed a significant improvement when we compared pre- and post-treatment values $(\mathrm{p}=0.05$ and $\mathrm{p}=0.04$ respectively). This difference became more striking when we considered the changes in FVC during the first 12 months of treatment $(\mathrm{p}=0.02)$. In particular, the 6MWT showed an improvement of an average of 53.2 meters after treatment (Fig. 4E), while the NSAA increased a mean of four points (Fig. 4F) and, more importantly, the mean FVC after treatment was $5 \%$ greater (Fig. $4 \mathrm{G}$ ).

We had previously shown that mRNA and protein levels of GDF-15 and FGF-21 correlate strongly and significantly in children with a range of mitochondrial diseases and in myogenic cell lines in which mitochondrial damage was induced experimentally ${ }^{12}$. In keeping with this previous result, baseline and longitudinal concentrations of both factors also correlated with each other significantly in treated patients $(\mathrm{p}<0.001$; Spearman $\mathrm{r}=0.843)$, (Fig. $4 \mathrm{H})$.

When analysing group 1 and group 2 together, basal circulating GDF-15 and FGF-21 concentrations correlated significantly and negatively with age-at-treatment initiation $(\mathrm{p}=0.006, \mathrm{r}=-0.7$ and $\mathrm{p}=0.004, \mathrm{r}=-0.7$ respectively) and with weight expressed as BMI $(\mathrm{p}<0.001, \mathrm{r}=-0.8$ and $\mathrm{p}=0.024$ and $\mathrm{r}=-0.5$ respectively). In addition, basal GDF-15 concentrations correlated with the meters walked in the $6 \mathrm{MWT}(\mathrm{p}=0.003, \mathrm{r}=-0.75)$.

Inflammatory cytokines profile in patients with TK2 deficiency. Mitochondrial diseases and, in particular, TK2 deficiency are often associated with local (skeletal muscle) inflammatory responses which may be related to muscle cell damage or necrosis ${ }^{12}$. Given that GDF-15 can be induced by various stimuli related to inflammation, we assessed the circulating levels of the inflammatory cytokines IL-1, IL-6, IL-8, IL-15 and TNF (before and after treatment) in the eight patients from group 2 with late-onset phenotype that were followed in hospitals in Spain. We did not observe a significant increase in the average basal levels of any of the cytokines in either group of patients relative to the levels in age-matched controls nor a consistent pattern of change with time in treated patients (Supp. Fig. 2). 

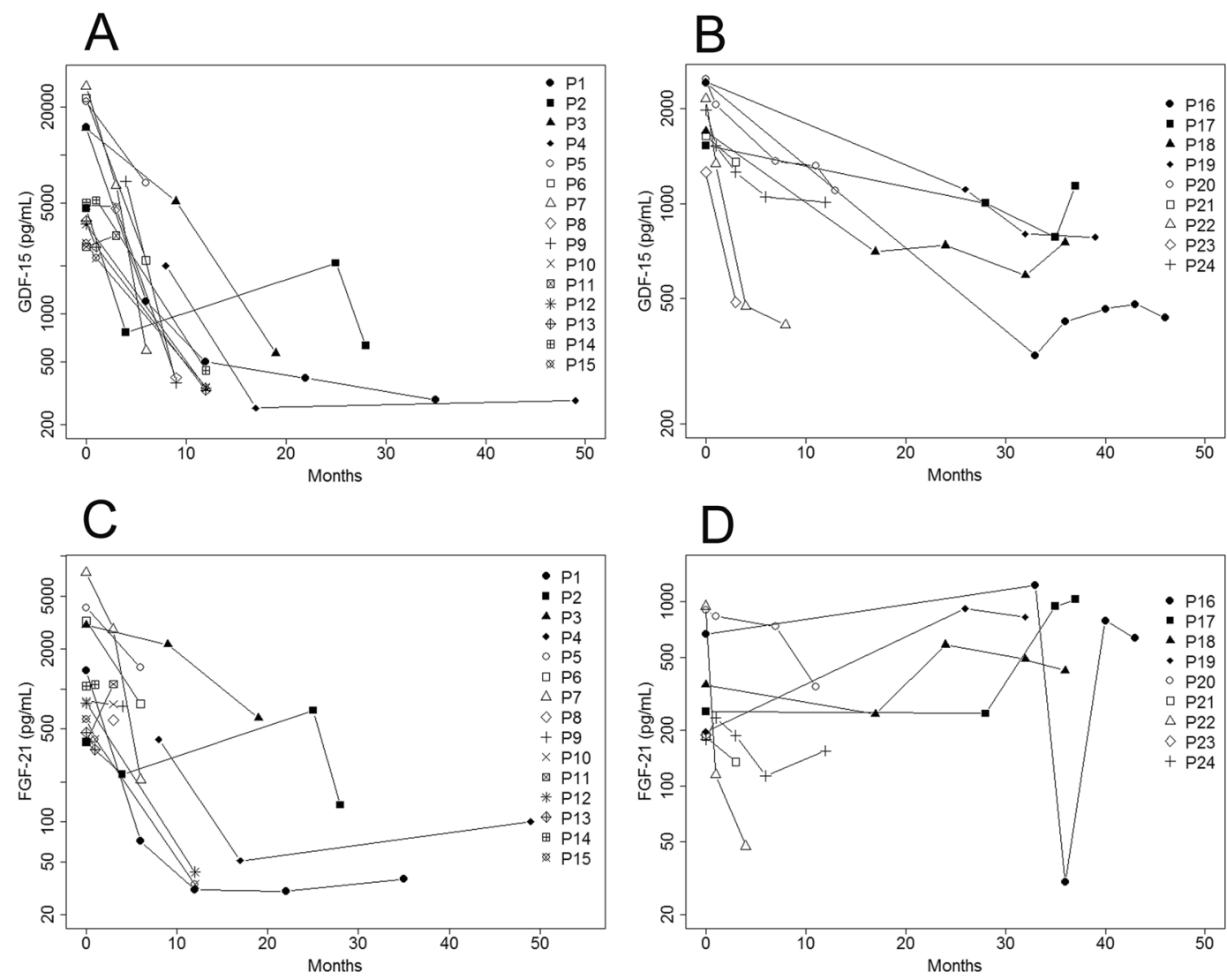

Figure 2. GDF-15 levels decreased during treatment with deoxynucleosides. Development of GDF-15 (A,B) and FGF-21 (C,D) circulating concentrations $(\mathrm{pg} / \mathrm{mL})$ over time in group 1 (left) and group 2 (right) patients.

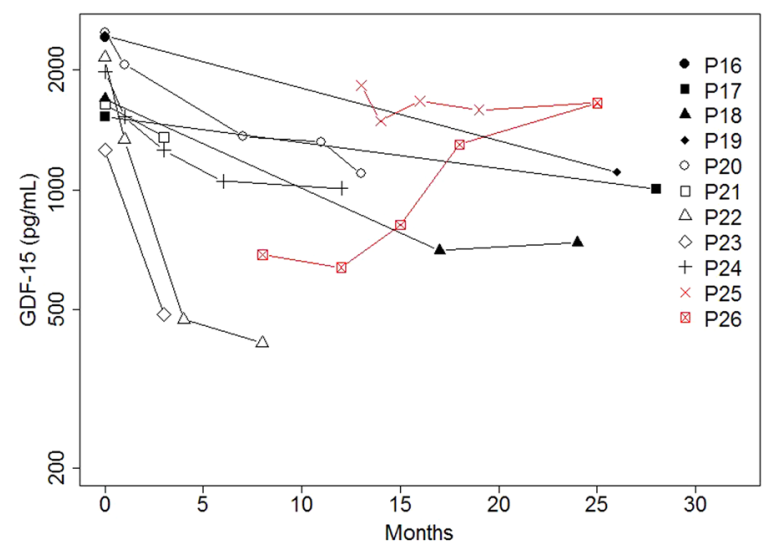

Figure 3. GDF-15 levels do not decrease over time in the absence of treatment. Development of serum GDF-15 over 28 months in treated patients (black lines) from group 2 versus untreated patients (P25 and P26, red lines).

\section{Discussion}

In previous work, our group described the global gene expression profile of skeletal muscle from patients with TK2 deficiency and identified the main molecular pathways implicated in the disease including the induction of GDF-15 expression partly under the control of p53. In addition, we demonstrated that GDF-15 outperformed other diagnostic biomarkers for mitochondrial diseases and postulated that it may also serve to monitor response to treatment based on preliminary data of one patient with TK2 deficiency that was treated with deoxynucleotides replacement therapy ${ }^{14,15}$. Since then, an expanded access program has shown a favorable side-effect profile and clinical efficacy of dNMP and deoxynucleoside therapies in patients with TK2 deficiency, some of them included in this study. Without any major side effects, the therapy had striking effects on early-onset severe myopathy patients, considerable functional improvements in childhood-onset cases and at least stabilization in late-onset ones ${ }^{4}$. 

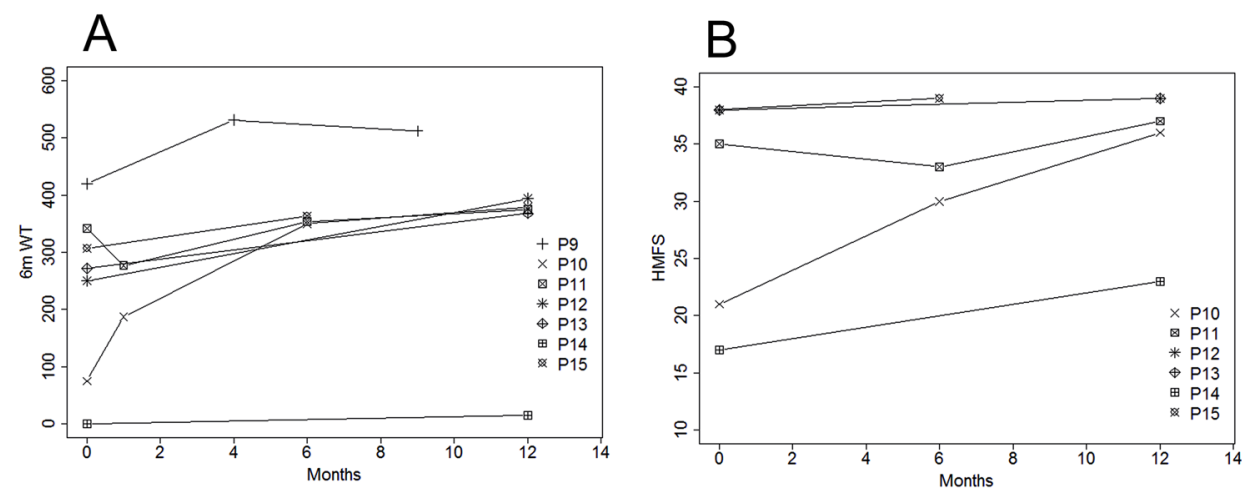

C

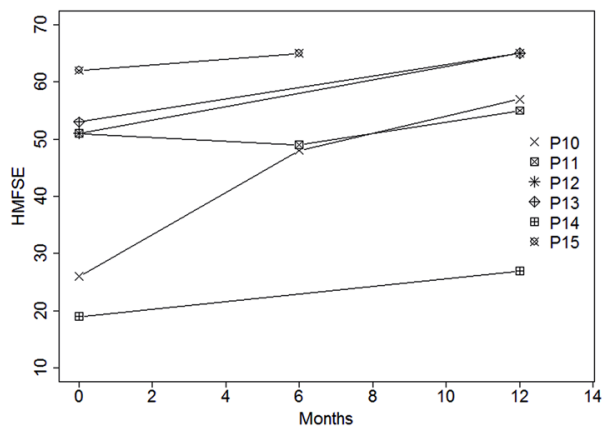

$\mathrm{D}$

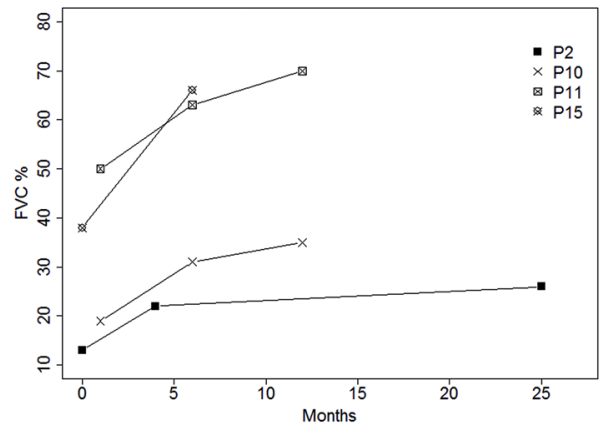

E
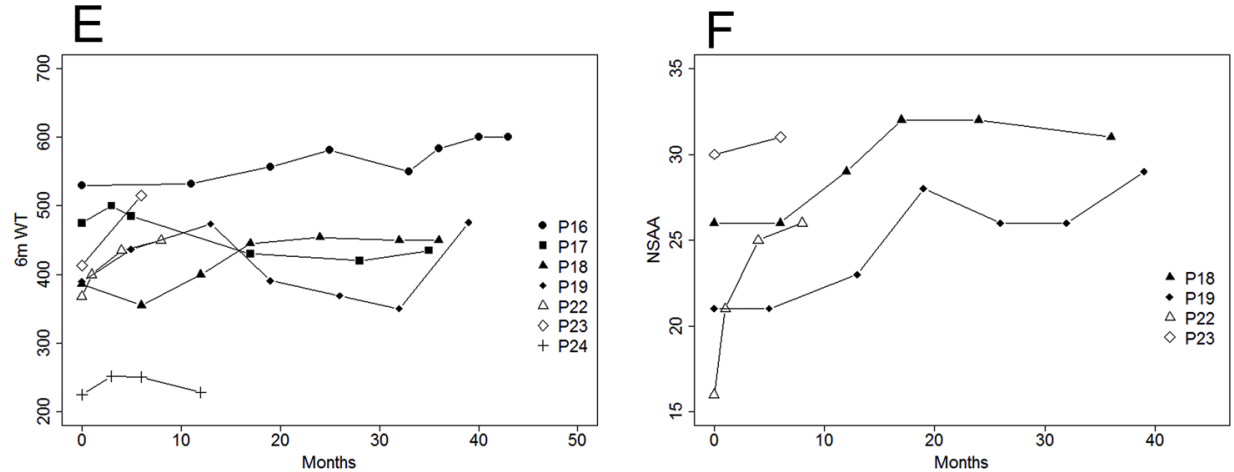

G
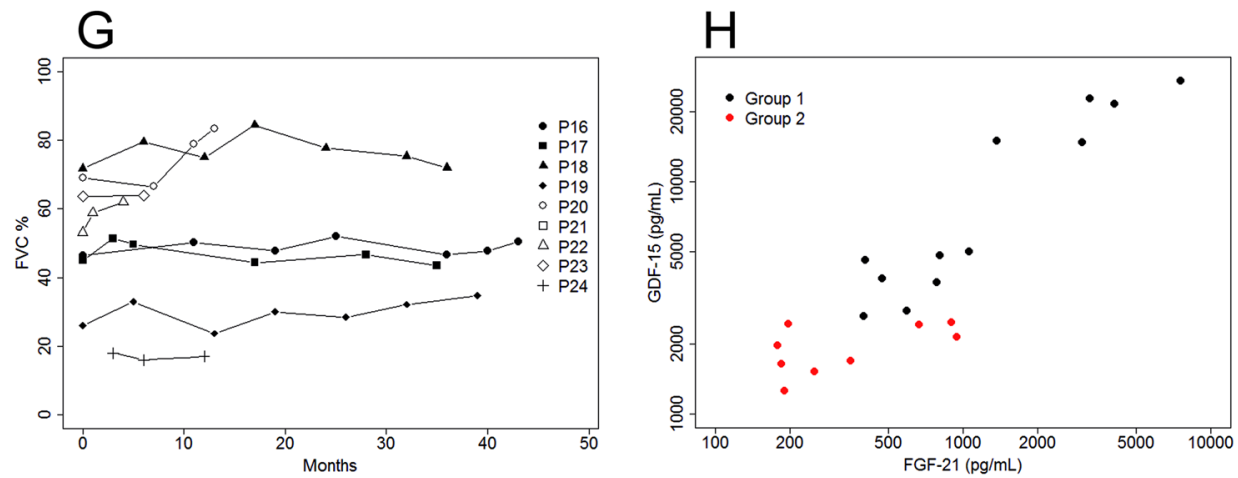

Figure 4. GDF-15 decrease was accompanied by improvement of several clinical outcome measures in treated patients. Development of selected functional tests for patients in group 1: 6MWT (A), HMFS (B), HMFSE (C), and \% FVC (D) and for patients in group 2: 6MWT (E), NSAA (F), and \% FVC (G) during treatment with deoxynucleosides. Correlation between basal levels of GDF-15 and FGF-21 in serum for group 1 (black) and group 2 (red) patients together $(\mathbf{H})$.

In the present work, we have assessed GDF-15 and FGF-21 in 24 patients with TK2 deficiency that started deoxynucleoside therapy either in their earlier years or as adults. The objective of this study was to ascertain the value of GDF-15 as a prognostic and treatment response biomarker for TK2 deficiency and compare it to FGF-21.

Together, the two groups of patients we analyzed represent the majority of patients with TK2 deficiency around the world that are treated. In addition, they encompass the known spectrum of the disease, from patients with 
early-onset and very rapid progression that is fatal in a few years without treatment, to childhood or late-onset cases with variable rates of disease progression, but also with bad prognosis because of respiratory involvement.

We confirm our previous findings that GDF-15 is markedly elevated in children and adults with TK2 deficiency $^{14,15}$. GDF-15 levels before the start of treatment were elevated above the normal threshold value in all patients and on average six times higher in the paediatric patients compared to the adult patients, even more so in the children with early-onset and the most severe form of the disease. Similar results were obtained for FGF- 21. Thus, both GDF-15 and FGF-21 are useful indicators of disease severity.

This was reflected in a significant and negative correlation between the basal concentration of both factors and the age of onset and/or treatment initiation so that the younger the children the higher the elevation of GDF-15 and FGF-21. Furthermore, pre-treatment GDF-15 levels were negatively associated with the meters walked on the $6 \mathrm{MWT}$, one of the main indicators of motor function and also with body weight, expressed as the BMI. Thus, the higher the levels of GDF-15 (and FGF-21) the lower the BMI. Considering the evidence of the role of GDF-15 in the regulation of body weight and appetite ${ }^{21}$, one may speculate that the dramatically increased levels of circulating GDF-15 observed in these patients may be directly contributing to the rapid loss of body weight which is an important component of the disease but this requires additional studies.

Our results demonstrate that in patients treated with deoxynucleosides, GDF-15 concentrations decrease following a significant dependence over time and therefore GDF-15 fulfills the criteria for a treatment response biomarker. Although a similar trend was obtained for FGF-21 the decrease was not as consistent or as marked, particularly for patients in group 2. In contrast to treated patients, in TK2 deficient patients without treatment levels of GDF-15 tended to increase over short periods of time although more natural history data on untreated patients are necessary. All the patients under treatment showed important beneficial effects that are maintained over time or, at least, the stabilization of the disease. The response to treatment was greatest in group 1 (the paediatric group), but the mild motor improvements and respiratory stability shown in group 2 (the adult group) are a relevant clinical result as well since they show that treatment can reduce morbidity and mortality also in patients who started treatment in adulthood. In keeping with the extent of the clinical benefit, the rate of GDF-15 decrease was faster in children than in adults, in particular, in the most severe patients who are the ones that respond more markedly to the treatment in the form of extended survival, recovery from ventilatory support dependency, and regaining motor abilities. It has been recently reported that age-related metabolic changes in mice (namely increased deoxynucleoside catabolism and decreased anabolism with age) account, at least in part, for the limited efficacy of the treatment in this model ${ }^{8}$.

Both GDF-15 and FGF-21 are broad action cytokine/hormone that are altered at the same time in many disease states where they are routinely employed as diagnostic and prognostic biomarkers ${ }^{12,20-25}$. In particular, they are increasingly used individually or in combination as diagnostic biomarkers of mitochondrial diseases ${ }^{9,16,18}$.

In this study, we found that both GDF-15 and FGF-21 were elevated in all patients before deoxynucleoside treatment but that GDF-15 was more robust than FGF-21 to monitor changes over time in treated patients. Furthermore, GDF-15 correlated with motor function (6MWT) although this needs to be confirmed in a larger group of treated patients.

Our previous transcriptomic data of skeletal muscle indicates that p53 is the key regulator in a network of genes which are coordinately activated in response to TK2 deficiency leading to inflammation, activation of muscle cell death by apoptosis and induction of GDF-15 in muscle and serum. p53 plays a fundamental role in the control of the cell cycle, DNA repair and apoptosis in the nucleus and in mitochondria. Under certain conditions such as increased oxidative stress, p53 translocates to mitochondria to repair and/or promote replication of mtDNA $^{14}$. Thus, our hypothesis is that mitochondrial DNA depletion due to TK2 mutations lead to activation of p53 which binds to the promoter region of GDF-15 inducing its expression and release by affected tissues.

Both GDF-15 and FGF-21 have a role in inflammation, for example, in pancreas, heart and adipose tissue $^{13,21-23}$. Our data show that the profile of inflammatory cytokines was not consistently altered at baseline (not even in the children with the most severe early-onset phenotype) indicating that the elevated levels of GDF-15 and FGF-21 are not secondary to systemic inflammation. Similarly, treatment with deoxynucleosides did not result in a consistent pattern of change on the baseline levels of the inflammatory cytokines. However, we cannot exclude a contribution from local, tissue-specific, inflammation since we have previously observed over-expression of inflammatory markers in muscle biopsies from patients with TK2 deficiency and other mitochondrial DNA depletion syndromes ${ }^{14}$.

Limitations of the current study were that biochemical and clinical evaluations were performed at different time intervals depending on where the patients were being followed and that functional scales were not uniform across the cohort. This was difficult to overcome since in some cases samples had been collected retrospectively and not as part of a controlled study and some patients started treatment several years ago. Another important limitation is that we did not have the possibility to follow the levels of GDF-15 and FGF-21 in a group of untreated patients with TK2 deficiency in order to find out how they would change over time. TK2 deficiency is a very rare disease and given the severity of the phenotype and the effectiveness of deoxynucleoside treatment the vast majority of patients worldwide are being treated.

In conclusion, we propose that GDF-15 may be a potential biomarker of disease severity and response to treatment with deoxynucleosides in patients with TK2 deficiency and potentially also in other forms of mitochondrial DNA depletion and deletion syndromes where this treatment may also be beneficial.

\section{Materials and Methods}

Study design. We determined circulating GDF-15 and FGF-21 at baseline and at various time points in a group of 26 patients with mutations in TK2 treated with deoxynucleosides. We examined the rate of change over time, and correlated its basal and follow-up levels with various clinical parameters related to disease severity and response to treatment. 
Standard protocol approvals, registrations, and patient consents. This study was conducted in accordance with the Declaration of Helsinki and legal regulations and was approved by the Ethics and Research Committee of the Fundación Sant Joan de Déu. Written informed consent was obtained from all patients or their parents/guardians prior to enrolment.

Study population and setting. Patients were included if they had two mutations in the TK2 gene and were receiving treatment with deoxynucleosides. Several of these patients have been described elsewhere ${ }^{9}$ and their basal characteristics are detailed in Table 1 and Supplementary Table S1. Patients were followed in five Hospitals in Spain (Hospital 12 de Octubre, Madrid; Hospital Sant Joan de Déu, Barcelona; Hospital de la Santa Creu I Sant Pau, Barcelona; Hospital Virgen del Rocio, Seville and Hospital Universitario Donostia, San Sebastian) and in the Columbia University Medical Center (New York, USA).

Oral administration of deoxynucleosides. All patients were treated with oral doses between 300$400 \mathrm{mg} / \mathrm{Kg} /$ day of each nucleoside (thymidine, dThd, and deoxycytidine, dCtd). Two patients (P3 and P4) started with nucleotides (dTMP and dCMP) and later switched to nucleosides (in a one-to-one ratio of height:weight), once it became clear that these are the active agents $s^{5,6}$. The majority of patients $(n=24)$ are currently being treated with dThd and dCtd. Two adult-onset individuals (P25 and P26) had to stop treatment because of liver enzymes elevation and were excluded from the main analyses.

Analytical methods. Serum samples were collected, allowed to clot, centrifuged at $1300 \mathrm{~g}$ for 10 minutes, aliquoted, and stored at $-80^{\circ} \mathrm{C}$ until the moment of the analysis. Long-term storage of samples at $-80^{\circ} \mathrm{C}$ does not affect GDF-15 or FGF-21 stability according to our experience and published data ${ }^{20}$.

Previously, we had compared serum (collected in serum separator tubes) and plasma samples (collected in EDTA, heparin lithium or heparin sodium tubes) from the same individuals and found comparable GDF-15 and FGF-21 levels regardless of the type of sample and collection method. We also compared different times of sample collection (fasting versus postprandial) and found no significant differences for either GDF-15 or FGF-21 (data not shown).

Quantitative sandwich ELISAs were performed using the Quantikine Human GDF-15 Immunoassay kit (R\&D Systems, Minneapolis, USA) and the FGF-21 ELISA KIT (R\&D Systems, Minneapolis, USA or Millipore, Massachusetts, USA) as previously described ${ }^{9,15}$. Sensitivity, intra-assay and inter-assay variances (expressed as $\mathrm{CV} \%$ ) are $4.39 \mathrm{pg} / \mathrm{mL}$ and $8.69 \mathrm{pg} / \mathrm{mL}, 2,26 \%$ and 3,43\% and 5,43 and 7,5\% for GDF-15 and FGF-21 respectively.

Briefly, serum samples were either diluted $1 / 4$ in the sample diluent buffer provided with the kit as recommended by the manufacturer (GDF-15) or used undiluted (FGF-21). A volume of 50ul of diluted serum sample was added to each well and assays were performed in duplicate. A standard curve was prepared using a dilution series (in pg/mL) of a recombinant human GDF-15 or FGF-21 standard diluted in the sample diluent buffer provided with the kit. To determine the optical density of the preparations we used a microplate reader (Molecular Probes) set to $450 \mathrm{~nm}$ subtracting readings at $540 \mathrm{~nm}$ for wavelength correction. The values from each sample were extrapolated from a standard four-parameter logistic curve using the SoftMax software of the microplate reader. Final concentrations were corrected by the dilution factor.

Interleukin (IL)-1B, IL-6, IL-8, IL-15, tumor necrosis factor (TNF) and monocyte chemoattractant protein-1 (MCP1) were quantified using a multiplex analysis system based on fluorescently labeled microspheres linked to specific antibodies (HCYTOMAG-60K-06, Millipore Linco Research/Millipore, Massachusetts, USA) using a Luminex100ISv2 instrument.

Outcome measures. Motor assessment: Patients underwent periodic motor assessments with at least one of the following: 6-minute walk test (6MWT); North Star Ambulatory Assessment (NSAA), which evaluates motor goals with a score range of $0-34$ as values of minimum and maximum motor skills, respectively; Egen Klassifikation (EK2), which evaluates functional capacity in nonambulatory patients with a score range of 30-0 as values of minimum and maximum functional capacity, respectively; the Children's Hospital of Philadelphia Infant Test of Neuromuscular Disorders (CHOP INTEND) used to evaluate the motor skills of infants with SMA with a score range from 0 to 64, with higher scores indicating better motor function; Hammersmith Motor Functional Scale (HFMS), which assesses the physical abilities of children with non-ambulant Spinal Muscular Atrophy, with a total score achievable of 40, with higher scores reflecting better physical abilities; or the Hammersmith Motor Functional Scale Expanded (HFMSE), which contains additional items for the ambulant population, with a total score achievable of 66.

Respiratory evaluation: We measured forced vital capacity (FVC) and maximal inspiratory pressure (MIP) in an upright position in compliant patients.

Statistical analysis. Descriptive statistics and correlation analyses were performed using R version 3.2 and GraphPad PRISM version 8.1.2 A. A p-value of $<0.05$ was considered statistically significant. Spearman's correlation was used to determine the relationship between numerical variables, and a paired non-parametric test was used to compare variables at two times (pre- versus post-treatment) for the same patient. Repeated measures mixed linear models were used to determine whether numerical variables changed over time if data was available at multiple time points.

\section{Data availability}

The raw data and protocols used for this study are available from the corresponding author.

Received: 22 January 2020; Accepted: 13 May 2020;

Published online: 22 June 2020 


\section{References}

1. Viscomi, C. \& Zeviani, M. MtDNA-maintenance defects: syndromes and genes. J. Inherit. Metab. Dis. 40, 587-599 (2017).

2. Garone, C. et al. Retrospective natural history of thymidine kinase 2 deficiency. J. Med. Genet. 55, 515-521 (2018).

3. Wang, J. et al. Clinical and molecular spectrum of thymidine kinase 2-related mtDNA maintenance defect. Mol. Genet. Metab. 124, 124-130 (2018)

4. Domínguez-González, C. et al. Late-onset thymidine kinase 2 deficiency: a review of 18 cases. Orphanet J. Rare Dis. 14, 100 (2019).

5. Lopez-Gomez, C. et al. Deoxycytidine and Deoxythymidine Treatment for Thymidine Kinase 2 Deficiency. Ann. Neurol. 81, 641-652 (2017).

6. Cámara, Y. et al. Administration of deoxyribonucleosides or inhibition of their catabolism as a pharmacological approach for mitochondrial DNA depletion syndrome. Hum. Mol. Genet. 23, 2459-67 (2014).

7. Lopez-Gomez, C. et al. Bioavailability and cytosolic kinases modulate response to deoxynucleoside therapy in TK2 deficiency. EBioMedicine 46, 356-367 (2019).

8. Blázquez-Bermejo, C. et al. Age-related metabolic changes limit efficacy of deoxynucleoside-based therapy in thymidine kinase 2-deficient mice. EBioMedicine 46, 342-355 (2019).

9. Domínguez-González, C. et al. Deoxynucleoside Therapy for Thymidine Kinase 2-Deficient Myopathy. Ann. Neurol. 86, 293-303 (2019).

10. Aartsma-Rus, A. \& Arechavala-Gomeza, V. Why dystrophin quantification is key in the eteplirsen saga. Nat. Rev. Neurol. 14, 454-456 (2018)

11. Kempf, T. et al. The transforming growth factor-beta superfamily member growth-differentiation factor- 15 protects the heart from ischemia/reperfusion injury. Circ. Res. 98, 351-60 (2006).

12. Chung, H. K. et al. Growth differentiation factor 15 is a myomitokine governing systemic energy homeostasis. J. Cell Biol. 216, 149-165 (2017).

13. Adela, R. \& Banerjee, S. K. GDF-15 as a Target and Biomarker for Diabetes and Cardiovascular Diseases: A Translational Prospective. J. Diabetes Res. 2015, 1-14 (2015).

14. Kalko, S. G. et al. Transcriptomic profiling of TK2 deficient human skeletal muscle suggests a role for the p53 signalling pathway and identifies growth and differentiation factor-15 as a potential novel biomarker for mitochondrial myopathies. BMC Genomics $\mathbf{1 5}$ (2014).

15. Montero, R. et al. GDF-15 Is Elevated in Children with Mitochondrial Diseases and Is Induced by Mitochondrial Dysfunction. PLoS One 11, e0148709 (2016).

16. Davis, R. L., Liang, C. \& Sue, C. M. A comparison of current serum biomarkers as diagnostic indicators of mitochondrial diseases. Neurology 86, 2010-2015 (2016).

17. Suomalainen, A. et al. FGF-21 as a biomarker for muscle-manifesting mitochondrial respiratory chain deficiencies: a diagnostic study. Lancet Neurol. 10, 806-818 (2011).

18. Yatsuga, S. et al. Growth differentiation factor 15 as a useful biomarker for mitochondrial disorders. Ann. Neurol., https://doi. org/10.1002/ana.24506 (2015).

19. Unsicker, K., Spittau, B. \& Krieglstein, K. The multiple facets of the TGF- $\beta$ family cytokine growth/differentiation factor-15/ macrophage inhibitory cytokine-1. Cytokine Growth Factor Rev. 24, 373-384 (2013).

20. Hamon, S. M. et al. Defining reference intervals for a serum growth differentiation factor-15 (GDF-15) assay in a Caucasian population and its potential utility in diabetic kidney disease (DKD). Clin. Chem. Lab. Med. 57, 510-520 (2019).

21. Baek, S. J. \& Eling, T. Growth differentiation factor 15 (GDF15): A survival protein with therapeutic potential in metabolic diseases. Pharmacol. Ther. 198, 46-58 (2019).

22. Kharitonenkov, A. \& DiMarchi, R. Fibroblast growth factor 21 night watch: advances and uncertainties in the field. J. Intern. Med. 281, 233-246 (2017).

23. Planavila, A., Fernández-Solà, J. \& Villarroya, F. Cardiokines as Modulators of Stress-Induced Cardiac Disorders. Adv. Protein Chem. Struct. Biol. 108, 227-256 (2017).

24. Tezze, C., Romanello, V. \& Sandri, M. FGF21 as Modulator of Metabolism in Health and Disease. Front. Physiol. 10, 419 (2019).

25. Planavila, A. et al. Fibroblast growth factor 21 protects the heart from oxidative stress. Cardiovasc. Res. 106, 19-31 (2014).

\section{Acknowledgements}

We wish to thank the patients and their families for their collaboration in this study. We are grateful to the Biobank of the Hospital Sant Joan de Déu (Spanish Biobank Network, ISCIII). We are grateful to Esther van de Vosse from EVScienceConsultant for editing the Manuscript. This work was supported by research grants of Plan Nacional de I + D + I and Instituto de Salud Carlos III (ISCIII), Subdirección General de Evaluación y Fomento de la Investigación Sanitaria, projects PI16/01843 (CP), PI16/00579 and CP09/00011 (CJM), PI15/00431 (MAM), PI17/00109 (RA) and PI18/01574 (RM), co-funded with European Regional Development Fund (ERDF) "A way to achieve Europe"; multicentric grant funded by the ISCIII (PMP15/00025, MAM, RM, CP), Grants of the Ministerio de Ciencia, Innovación y Universidades SAF2017-85722 (FV) and SAF2017-87506-R (YC), the Muscular Dystrophy Association grant 577391 (MH), and the Arturo Estopinan TK2 Research Fund (MH).

\section{Author contributions}

C.D.G., A.B., M.H., A.N., C.J.M. contributed to the conception and design of the work, acquisition, analysis and interpretation of data and drafting the work or substantively revised it. C.B., M.M.G., I.M., C.P., C.O., J.D.M., J.A.P., S.T., D.C., S.G.K., C.B.B., Y.C., R.M., F.M.S., M.A.M., J.M., E.R.P., J.V., R.M., F.V., R.A. contributed to the analysis and interpretation of data and revising the work. All authors have approved the submitted version of this M.S.

\section{Competing interests}

MH, RM, Columbia University Medical Center (CUMC), and the Vall d'Hebron Research Institute (VHIR) have filed patent applications for deoxynucleoside and deoxynucleotide therapies for human mitochondrial DNA depletion and deletions syndrome including TK2 deficiency. RM, YC, CB, VHIR, and The Biomedical Network Research Centre on Rare Diseases (CIBERER) have filed patent applications covering potential use of deoxynucleoside treatment for POLG deficiency and other mtDNA replication defects in humans. CUMC, VHIR, and CIBERER have licensed pending patent applications related to these technologies to Modis Therapeutics, Inc. CUMC, VHIR, and CIBERER may be eligible to receive payments related to the development and commercialization of the technologies. Any potential licensing fees earned will be paid to CUMC, VHIR, 
and CIBERER and are shared with all inventors mentioned above through VHIR and CIBERER policies on distribution and objectivity in research. MH and RM serve as paid consultants to Modis Therapeutics, Inc. and RM has equity in this company. RM, YC, CB, VHIR, and The Biomedical Network Research Centre on Rare Diseases (CIBERER) have filed patent applications covering the potential use of deoxynucleoside treatment as a way to increase mtDNA copy number.

\section{Additional information}

Supplementary information is available for this paper at https://doi.org/10.1038/s41598-020-66940-8.

Correspondence and requests for materials should be addressed to C.J.-M.

Reprints and permissions information is available at www.nature.com/reprints.

Publisher's note Springer Nature remains neutral with regard to jurisdictional claims in published maps and institutional affiliations.

(c) (i) Open Access This article is licensed under a Creative Commons Attribution 4.0 International License, which permits use, sharing, adaptation, distribution and reproduction in any medium or format, as long as you give appropriate credit to the original author(s) and the source, provide a link to the Creative Commons license, and indicate if changes were made. The images or other third party material in this article are included in the article's Creative Commons license, unless indicated otherwise in a credit line to the material. If material is not included in the article's Creative Commons license and your intended use is not permitted by statutory regulation or exceeds the permitted use, you will need to obtain permission directly from the copyright holder. To view a copy of this license, visit http://creativecommons.org/licenses/by/4.0/.

(c) The Author(s) 2020 